\title{
APRENDIZAGEM DE RELAÇÕES PALAVRA-OBJETO POR EXCLUSÃO EM CRIANÇAS TÍPICAS E COM ATRASO DA FALA
}

\section{LEARNING OF WORD-OBJECT RELATIONS IN LATE TALKERS AND TYPICALLY DEVELOPING CHILDREN}

\author{
FeRnANDA MARQues ReSENDE - ORCID 0000-0003-4512-5053 \\ Universidade Estadual Paulista Julio de Mesquisa Filho, Brasil
}

JÚLIA FONSECA - ORCID 0000-0002-0247-7334

FACULDADE DE CIÊNCIAS BIOMÉDICAS DE CACOAL, BRASIL

\author{
ANDRÉIA SCHMIDT - ORCID 0000-0002-8836-6618 \\ Universidade de São Paulo e Instituto Nacional de CiênCia e TeCnologia Sobre Comportamento, Cognição \\ E ENSINO, BRASIL
}

\begin{abstract}
RESUMO
Pesquisas afirmam que crianças com atraso da fala têm dificuldades em apresentar o responder por exclusão, processo relacionado à aprendizagem de vocabulário. O objetivo desta pesquisa foi comparar a ocorrência do responder por exclusão, a aprendizagem de duas relações nome-objeto e a nomeação desses objetos por crianças com desenvolvimento típico da linguagem (DT) e crianças com atraso da fala (AF), com idades entre 3 e 4 anos. Participaram 19 crianças (oito com AF). Na Fase 1, foi estabelecida uma linha de base de discriminações condicionais com objetos conhecidos; na Fase 2 foram apresentadas tentativas de exclusão (consequenciadas diferencialmente) para dois objetos desconhecidos, entre as tentativas de linha de base; na Fase 3 foram realizadas sondas de nomeação e de emparelhamento ao modelo: a criança deveria nomear os dois objetos desconhecidos e seleciona-los (entre outros objetos disponíveis) diante de seu nome ditado. Caso a criança não nomeasse e selecionasse corretamente os dois objetos desconhecidos, as Fases 2 e 3 eram reapresentadas, até o limite de 10 reexposições. Todas as crianças atingiram o critério estabelecido nas sondas de emparelhamento ao modelo em até seis reexposições, mas apenas seis crianças (três de cada grupo) atingiram o critério nas sondas de nomeação. Não foram encontradas diferenças entre as crianças DT e AF nos desempenhos avaliados. A quantidade de exposição às novas relações para ocorrência de nomeações corretas foi maior que o relatado pela literatura. Sugere-se que as dificuldades de ampliação de vocabulário de crianças com AF não podem ser atribuídas a dificuldades específicas no desempenho por exclusão.
\end{abstract}

Palavras-chave: responder por exclusão, aprendizagem de relações nome-objeto, atraso de fala, crianças.

\begin{abstract}
Some studies suggest that children with speech delay have difficulties in presenting responding by exclusion. The aim of this research was to compare the occurrence of the responding by exclusion and the learning of two nameobject relations by children with typical language development (TD) and children with speech delay (SD), aged between 3 and 4 years. Nineteen children participated (eight SD). In Phase 1, a baseline of conditional discriminations with known objects was established; in Phase 2, four exclusion trials (two undefined names) were presented among baseline trials; in Phase 3 naming and matching probes were performed: the child should name the two unknown objects and select them, among other available objects, in matching to sample trials. If the child did not correctly name and select the two unknown objects, Phases 2 and 3 were repeated, up to the limit of 10 repetitions. All the children reached the criteria established in the matching probes in up to six reexpositions, but only six children (three in each group) reached the criterion in the naming probes. No differences were found between TD and SD children. The results replicate data from the literature on the faster occurrence of learning of listener performances compared to the learning of speaker performances, but differs from other researches on the amount of exposure required for the occurrence of correct naming responses. Similar performances presented by both groups indicate that the difficulties of vocabulary expansion of children with SD cannot be attributed to specific difficulties in performance by exclusion.

Key words: responding by exclusion, learning of word-object relations, speech delay, children

\footnotetext{
Essa pesquisa faz parte do programa científico do Instituto Nacional de Ciência e Tecnologia sobre Comportamento, Cognição e Ensino (INCT-ECCE), do qual a segunda autora faz parte, financiado pelo Conselho Nacional de Desenvolvimento Científico e Tecnológico (CNPq, processo $n^{\circ}$ 465686/2014-1) e pela FAPESP (processo $n^{\circ}$ 2014/50909-8). Correspondência referente a este artigo devem ser dirigidas a Andréia Schmidt (Faculdade de Filosofia, Ciências e Letras de Ribeirão Preto (USP - FFCLRP), Departamento de Psicologia. E-mail: aschmidt@ffclrp.usp.br).
}

DOI. 10.18542/rebac.v14i1.7154 
De acordo com Ellis Weismer, Venker, Evans e Moyle (2013), crianças com atraso de fala (AF) são aquelas que demonstram habilidades de linguagem expressiva atrasadas, tipicamente identificadas por um déficit de vocabulário ou combinações limitadas de duas palavras, em comparação a crianças com desenvolvimento típico da linguagem (DT). Segundo Hage e Guerreiro (2010), crianças com atraso da fala são geralmente identificadas a partir dos dois anos de idade, com vocabulário pequeno e uma diferença de pelo menos 12 meses entre a idade cronológica e a idade linguística, não apresentando déficit neurológico, sensório ou cognitivo subjacentes. $O$ atraso da fala atinge cerca de $12 \%$ da população americana (e.g., Zubrick, Taylor, Rice, \& Slegers, 2007). No Brasil, apesar de serem registrados estudos sobre a prevalência de desvios fonológicos $(9,17 \%$ - Cavalheiro, Brancalione, \& Keske-Soares, 2012) e de desordens de fala envolvendo aspectos fonéticofonológicos (24,6\% - Goulart \& Chiari, 2007), não foram encontrados estudos específicos sobre prevalência de atrasos de fala.

O atraso da fala está correlacionado com dificuldades em habilidades de leitura e com baixo desempenho em uma ampla gama de habilidades de linguagem ao longo da infância (Rescorla, 2002), bem como a um menor nível de vocabulário e memória verbal na adolescência, em comparação a indivíduos com desenvolvimento típico (Rescorla, 2009). Esses dados indicam a necessidade de se estudar as variáveis que podem produzir o atraso da fala, bem como as características de desempenho dessas crianças ao longo do desenvolvimento da linguagem, com vistas a se planejar intervenções preventivas e remediativas, uma vez que elas apresentam riscos para problemas de linguagem (oral e escrita).

Uma das características de desempenho estudadas em crianças com atraso da fala é o responder por exclusão, referido como mapeamento rápido (fast mapping) por autores ligados à Psicologia Cognitiva (Eyer et al., 2002). Ellis Weismer et al. (2013) realizaram um estudo em que investigaram o responder por exclusão em 30 crianças com AF e em 32 crianças com DT, com idades entre 2 e 5 anos. A tarefa proposta ocorria em um contexto de brincadeira de piquenique, na qual brinquedos plásticos com forma de maçã e cookie, além de dois outros com formas indefinidas, que foram relacionados a palavras também indefinidas (koob e tade), eram colocados $\mathrm{e}$ retirados de uma cesta. $\mathrm{O}$ procedimento compreendia três fases. $\mathrm{Na}$ primeira (exposição), cada um dos quatro brinquedos era apresentado à criança simultaneamente ao seu respectivo nome: "Esse é um [koob]. Coloque-o na cesta". Na segunda fase (produção) cada brinquedo era retirado da cesta individualmente e mostrado para a criança: "O que é isso?". Finalmente, na terceira fase (compreensão) outros dois brinquedos plásticos com formas indefinidas foram incluídos na cesta (portanto, havia seis brinquedos para escolha) e a criança deveria selecionar um deles diante da instrução: "Pode me dar o [koob]?". Essa tarefa era considerada pelos autores como de mapeamento rápido - ou, como uma tarefa de responder por exclusão, uma vez que a criança deveria relacionar os brinquedos indefinidos aos seus respectivos nomes, também indefinidos. As crianças com AF obtiveram escores significativamente menores nas tarefas de compreensão e de produção de palavras (novas e familiares), em comparação às crianças com desenvolvimento típico. O estudo sugeriu, também, que crianças com AF apresentam um desempenho pior nas tarefas de responder por exclusão em comparação às crianças com DT. Apesar das evidências apresentadas, é importante destacar que o tipo de tarefa escolhido para mensurar o mapeamento rápido foi diferente aqui das tarefas utilizadas em estudos da Análise do Comportamento para avaliar o responder por exclusão, como será visto a seguir.

$\mathrm{O}$ responder por exclusão pode ser definido como a seleção de um estímulo de comparação indefinido (estímulo não relacionado previamente a qualquer estímulo modelo) diante de um estímulo modelo também indefinido (Costa, de Rose, \& de Souza, 2010; Dixon, 1977). Estudos experimentais sobre o responder por exclusão envolvem, inicialmente, o estabelecimento de uma linha de base de emparelhamento arbitrário com o modelo, em que a criança tem a oportunidade de relacionar figuras ou objetos conhecidos (estímulos de comparação) a palavras ditadas também conhecidas (estímulos modelo). Em seguida, ela é exposta a tentativas de sonda (testes ou sondas de exclusão), nas quais uma palavra desconhecida (e.g., "xipite") é apresentada como modelo, diante de estímulos de comparação conhecidos e de um estímulo novo como possibilidade de escolha. A criança estará respondendo por exclusão se relacionar a palavra nova ("xipite") ao estímulo de comparação também novo.

Apesar da literatura referir que a aprendizagem de novas palavras tem estreita relação com o responder por exclusão, diversos estudos têm demonstrado que um número reduzido de exposições a tentativas de responder por exclusão não é suficiente para que ocorra a aprendizagem de novas relações palavra-objeto (Domeniconi, Costa, de Rose, \& de Souza, 2007; Schmidt, Franco, Lotério, \& Gomes, 2016; Souza, Gil, \& Garcia, 2018; Souza, Sousa, \& Gil, 2016; Wilkinson \& McIlvane, 1997). Porém, a exposição repetida a tentativas similares às de responder por exclusão pode favorecer essa aprendizagem, mesmo em crianças com menos de 36 meses (Costa, Grisante, Domeniconi, de Rose, \& de Souza, 2013; Souza et al., 2016).

O número de exposições a tentativas de exclusão necessário para a aprendizagem de novas relações entre palavras-figuras/objetos tem sido estudado em pesquisas com indivíduos com desenvolvimento típico e atípico (Costa et al, 2013; Langsdorff, Domeniconi, Schmidt, Gomes, \& de Souza, 2017; Langsdorff, Schmidt, \& Domeniconi, 2015; Souza et al., 2016). Os estudos com esse objetivo têm procedimentos variados. Por exemplo, alguns são computadorizados (e.g., Costa et al., 2013), nos quais as tarefas de emparelhamento ao modelo são apresentadas na tela de um computador; outros são procedimentos "de mesa" (e.g., Langsdorff et al., 2017), 
em que a tarefa ocorre a partir da apresentação de modelo ditado e de figuras em cartões, ou em "cadernos de tentativas" como comparações; em outros procedimentos, o contexto é de brincadeira, em que os estímulos modelo são ditados, enquanto que brinquedos tridimensionais são dispostos no chão, em mesas ou outros aparatos e as tarefas são conduzidas em formato mais "lúdico" (e.g., Domeniconi et al., 2007; Souza et al., 2018). A tarefa básica, no entanto, é a mesma: após o estabelecimento de uma linha de base de relações nome-figura (ou nomeobjeto) conhecidas, um novo nome é ditado e um estímulo desconhecido é introduzido entre os estímulos de comparação; caso o responder por exclusão ocorra, o estímulo desconhecido é selecionado. A aprendizagem por exclusão é aferida quando, em tentativas posteriores, o mesmo nome desconhecido é ditado mas, entre os estímulos de comparação, além das figuras conhecidas e daquela figura previamente relacionada ao modelo, uma figura completamente nova também é apresentada. A tarefa da criança consiste em discriminar entre as figuras "desconhecidas", de forma que a seleção da figura previamente relacionada ao modelo ditado indicaria que a criança aprendeu a relação; a seleção da figura completamente nova indicaria que a resposta da criança estaria sob controle da novidade do estímulo, o que geraria a exposição a nova(s) tentativa(s) do tipo exclusão ${ }^{1}$, até que critérios de aprendizagem sejam atingidos. Além disso, é importante aferir a estabilidade dessa relação emergente ao longo do tempo, o que caracterizaria de forma mais clara a aprendizagem (Souza \& Gil, 2018).

A despeito de pequenas variações nesse formato básico (configuração das tentativas de sonda, por exemplo), as pesquisas têm indicado que o número médio de exposições a tentativas do tipo exclusão, necessário para a ocorrência de aprendizagem de novas relações, varia entre três e dez. Importante destacar que a maioria das pesquisas que investiga a aprendizagem a partir de tentativas de exclusão tem como medida de aprendizagem o desempenho de seleção da figura diante do nome, sem a sondagem do desempenho de nomeação (mas, ver Souza et al., 2016; e Costa et al., 2013, melhor descrito adiante).

Uma questão de interesse, e que tem sido investigada em algumas pesquisas, é o processo de aprendizagem simultânea de duas relações nomeobjeto/figura por exclusão. Wilkinson, Ross e Diamond (2003) compararam a aprendizagem de duas novas relações palavra-objeto em crianças de 26 a 54 meses de idade em duas condições: introdução concorrente de

\footnotetext{
${ }^{1}$ Cabe, aqui, uma explicação. A rigor, o responder por exclusão ocorreria apenas na primeira exposição ao modelo e comparação desconhecidos (Dixon, 1977), como uma relação emergente. As exposições posteriores não poderiam ser chamadas de "tentativas de exclusão" em função da história prévia de exposição à relação. Além disso, essas tentativas posteriores, se programadas com consequências diferenciais para os desempenhos dos participantes, são melhor referidas como tentativas de ensino. Para fins de simplificação do relato, porém, dar-se-á o nome de "tentativas do tipo exclusão" para aquelas tentativas que envolvem a reexposição do participante à relação modelo-comparação indefinidos, com ou sem consequências diferenciais para os desempenhos.
}

estímulos e introdução sucessiva de estímulos. $\mathrm{Na}$ introdução concorrente de estímulos, uma palavra desconhecida (p1) era ditada diante de três possibilidades de escolha: dois brinquedos conhecidos e um novo (b1). $\mathrm{Na}$ tentativa seguinte, outra palavra desconhecida (p2) era ditada e outro brinquedo novo (b2) estava disponível entre os conhecidos. As palavras novas se alternavam nas tentativas e só o brinquedo novo correspondente a cada uma estava disponível para a escolha, entre os conhecidos. No procedimento de introdução sucessiva de estímulos, a apresentação da primeira palavra nova (p1) e do primeiro brinquedo novo (b1) era idêntica à da introdução concorrente de estímulos. Porém, a apresentação da segunda palavra nova (p2) e do segundo brinquedo novo (b2) era feita pela inclusão de b2 junto a b1, que acabara de ser selecionado na tentativa anterior, além de outros brinquedos conhecidos. Para ambas as condições, os testes de verificação de aprendizagem foram iguais: as palavras novas se alternavam como modelos e os dois objetos novos estavam disponíveis entre os familiares para a escolha. As crianças mais jovens (até 42 meses) aprenderam melhor as novas palavras na condição de introdução sucessiva pois, apesar de o procedimento ter se revelado inicialmente mais difícil para elas, aparentemente a fase de ensino as preparava melhor para os testes com a presença simultânea dos dois brinquedos novos. Neste estudo, porém, foram feitas apenas três exposições de cada nova relação, o que se mostrou insuficiente para uma aprendizagem estável das relações nome-objeto.

Ribeiro e Schmidt (2015) replicaram o procedimento de introdução sucessiva de estímulos proposto por Wilkinson et al. (2003) com sete bebês de 17 a 22 meses, mas aumentaram o número de exposições às palavras novas de três para cinco. Apesar de ter-se verificado indícios de aprendizagem das relações nomeobjeto em dois dos bebês, os demais tiveram seu desempenho de linha de base perturbado pela condução de tentativas de sonda em extinção.

Costa et al. (2013) realizaram um estudo com oito crianças entre 4 e 5 anos com o objetivo investigar a quantidade de exposições necessárias para que as crianças nomeassem duas figuras novas emparelhadas, por exclusão, a palavras novas, em um procedimento computadorizado. O procedimento de ensino empregado foi semelhante ao de introdução concorrente de estímulos (Wilkinson et al., 2003). As sondas de aprendizagem envolviam a apresentação da figura-alvo junto a uma figura completamente desconhecida e um comparação vazio (uma "máscara" introduzida gradualmente, que poderia se sobrepor a qualquer um dos estímulos de comparação ou a nenhum deles - Wilkinson \& McIlvane, 1997), ou a apresentação de uma figura conhecida, uma completamente desconhecida e a máscara. O critério de aprendizagem (nomeação dos dois estímulos novos num mesmo bloco de tentativas) foi atingindo depois de no mínimo três exposições (um participante), e no máximo dez exposições às tentativas do tipo exclusão, com média de seis exposições.

O conjunto desses estudos mostra que o número de exposições a tentativas de exclusão é relevante para a 
ocorrência da aprendizagem de relações nomeobjeto/figura, tanto em tarefas que envolvem a seleção da figura ou do objeto, quanto em tarefas de nomeação. Considerando que a literatura aponta indícios, ainda que incipientes, de que crianças com AF têm dificuldades em apresentar desempenho por exclusão e que ainda não se sabe se essa suposta dificuldade acarretaria na necessidade de maior exposição a tentativas do tipo exclusão para a aprendizagem de relações nome-objeto desconhecidas, o presente estudo teve como objetivo comparar a ocorrência do responder por exclusão e a aprendizagem de duas relações nome-objeto por crianças com DT e crianças com $\mathrm{AF}$, com idades entre 3 e 4 anos. A hipótese do estudo é que crianças com atraso da fala precisariam de um número maior de exposições às tentativas do tipo exclusão com reforço diferencial para aprenderem as relações nomeobjeto (selecionarem o objeto "desconhecido" correspondente à palavra nova, e nomearem esse objeto), em comparação com crianças com desenvolvimento típico.

\section{Participantes}

\section{MÉTODO}

Participaram 19 crianças (12 meninos), com idade entre 3 e 4 anos, todas frequentadoras de uma creche filantrópica da cidade de Ribeirão Preto - SP. Onze das 19 crianças apresentaram desenvolvimento típico da linguagem (DT - P1 a P11, todos com vocabulário auditivo e expressivo médio) e oito apresentaram atraso da fala (AF - P12 a P19, todos com vocabulário auditivo médio e vocabulário expressivo muito rebaixado). A caracterização individual dos participantes pode ser verificada na Tabela 1. A participação das crianças foi autorizada pelos pais por meio de assinatura do Termo de Consentimento Livre e Esclarecido e a pesquisa foi aprovada pelo Comitê de Ética em Pesquisa com Seres Humanos da Faculdade de Filosofia, Ciências e Letras de Ribeirão Preto - USP (CAAE n ${ }^{\circ}$ 37558314.7.0000.5407).

\section{Materiais e Equipamentos}

Foram utilizados objetos familiares (definidos) brinquedos cujos nomes eram conhecidos pelas crianças: bola, carrinho e as pelúcias de um sapo, um cachorro e um macaco. A familiaridade desses objetos foi verificada previamente, apresentando-os para três crianças de mesma idade (que frequentavam a mesma escola dos participantes) e que não participaram da pesquisa: todas nomearam corretamente esses objetos. Foram empregados também objetos não familiares (indefinidos) - brinquedos construídos pelas pesquisadoras e que não tinham sido relacionados com qualquer nome conhecido na história da criança (ver Tabela 2, adiante). As palavras indefinidas empregadas (xipite e fulito) não têm significado formal na língua portuguesa e já foram empregadas em outros estudos similares ao aqui apresentado (e.g., Ribeiro \& Schmidt, 2015). As sessões foram filmadas com uma filmadora Sony Handycam HDR CX-220.

Tabela 1.

Idade, Gênero e Escores nos Testes de Vocabulário Auditivo e Expressivo dos Participantes.

\begin{tabular}{|c|c|c|c|c|}
\hline Participante & Idade & Gênero & Vocabulário Auditivo & $\begin{array}{l}\text { Vocabulário } \\
\text { Expressivo }\end{array}$ \\
\hline DT1 & $4 \mathrm{a}$ & $\mathrm{M}$ & 32 (médio) & 88 (médio) \\
\hline DT2 & $4 \mathrm{a} 3 \mathrm{~m}$ & M & 32 (médio) & 97 (muito elevado) \\
\hline DT3 & $4 \mathrm{a} 4 \mathrm{~m}$ & M & 30 (médio) & 93 (médio) \\
\hline DT4 & $4 \mathrm{a} 5 \mathrm{~m}$ & M & 31 (médio) & 88 (médio) \\
\hline DT5 & $4 \mathrm{a} 5 \mathrm{~m}$ & M & 32 (médio) & 90 (médio) \\
\hline DT6 & $4 \mathrm{a} 5 \mathrm{~m}$ & $\mathrm{~F}$ & 33 (médio) & 95 (médio) \\
\hline DT7 & $4 \mathrm{a} 6 \mathrm{~m}$ & $\mathrm{~F}$ & 31 (médio) & 89 (médio) \\
\hline DT8 & $4 \mathrm{a} 6 \mathrm{~m}$ & M & 32 (médio) & 89 (médio) \\
\hline DT9 & $4 \mathrm{a} 7 \mathrm{~m}$ & M & 31 (médio) & 85 (rebaixado) \\
\hline DT10 & $4 \mathrm{a} 7 \mathrm{~m}$ & $\mathrm{~F}$ & 33 (médio) & 91 (médio) \\
\hline DT11 & 4a $11 \mathrm{~m}$ & M & 33 (médio) & 88 (médio) \\
\hline AF12 & $3 \mathrm{a} 7 \mathrm{~m}$ & $\mathrm{~F}$ & 33 (médio) & 75 (muito rebaixado) \\
\hline AF13 & $3 \mathrm{a} 8 \mathrm{~m}$ & $\mathrm{~F}$ & 33 (médio) & 68 (muito rebaixado) \\
\hline AF14 & $3 \mathrm{a} 10 \mathrm{~m}$ & M & 29 (médio) & 68 (muito rebaixado) \\
\hline AF15 & $4 \mathrm{a} 1 \mathrm{~m}$ & M & 27 (médio) & 77 (muito rebaixado) \\
\hline AF16 & $4 \mathrm{a} 2 \mathrm{~m}$ & M & 29 (médio) & 68 (muito rebaixado) \\
\hline AF17 & $4 a 3 m$ & $\mathrm{~F}$ & 29 (médio) & 68 (muito rebaixado) \\
\hline AF18 & $4 \mathrm{a} 4 \mathrm{~m}$ & $\mathrm{~F}$ & 33 (médio) & 78 (muito rebaixado) \\
\hline AF19 & $4 \mathrm{a} 6 \mathrm{~m}$ & M & 27 (médio) & 68 (muito rebaixado) \\
\hline
\end{tabular}

Nota: $\mathrm{M}=$ masculino; $\mathrm{F}=$ feminino; $\mathrm{DT}=$ desenvolvimento típico da linguagem; $\mathrm{AF}=$ atraso da fala. 


\section{Instrumentos}

Para avaliar o desenvolvimento da linguagem dos participantes, foram utilizados o Teste de Vocabulário Auditivo Usp na forma abreviada original (TVAud-A33o) e o Teste de Vocabulário Expressivo na forma original (TVExp-100o) (Capovilla, Negrão, \& Damázio, 2011), padronizados para população brasileira, mas que não permitem classificação do vocabulário segundo faixas etárias; os testes avaliam se o desempenho da criança é adequado à idade, superior, rebaixado ou muito rebaixado.

O TVExp-100o é composto por um caderno de 100 páginas, cada qual contendo uma figura e a tarefa da criança consiste em nomear a figura mostrada pelo aplicador (uma tentativa por página). A pontuação varia de 1 a 100. Foram consideradas com atraso as crianças que apresentaram vocabulário expressivo classificado pelo teste como "muito rebaixado".

O TVAud-A33o é composto por 33 itens, apresentados em um caderno de sete páginas. As pranchas 1 a 6 contêm cinco itens cada, e a prancha 7 contém três itens. A tarefa da criança é apontar para a figura correspondente à palavra ditada pelo aplicador. A pontuação varia de 0 a 33 .

\section{Local e Situação experimental}

As sessões foram conduzidas em uma sala cedida pela escola, iluminada natural e artificialmente, com pouco ou nenhum ruído externo, na qual ficavam a pesquisadora e a criança. A sala tinha uma mesa de 1,60 $\mathrm{x} 0,70 \mathrm{~m}$ e seis cadeiras. A pesquisadora e a criança se sentavam no chão, uma em frente a outra. Entre elas, eram expostos horizontalmente e em linha alguns brinquedos (quatro por vez), lado a lado, sempre em sequência diferente a cada tentativa. Os brinquedos ficavam a aproximadamente $50 \mathrm{~cm}$ da criança. Cada tentativa iniciava com um pedido da pesquisadora para que a criança pegasse e lhe entregasse um dos brinquedos disponíveis (o nome ditado poderia ser definido ou indefinido). A tentativa se encerrava com a entrega do brinquedo à pesquisadora pela criança. Nas sondas de nomeação, cada brinquedo era apresentado individualmente para a criança e era pedido que ela dissesse o nome daquele brinquedo.

As sessões foram filmadas e as respostas dos participantes registradas pela pesquisadora durante a aplicação do procedimento, em folha de registro, e depois das sessões, a partir da transcrição das filmagens.

\section{Procedimentos}

As sessões foram conduzidas individualmente, uma vez por semana. Cada sessão teve duração de aproximadamente 20 minutos. No total, cada criança participou de três sessões: uma de familiarização com a tarefa e avaliação do repertório de linguagem e outras duas do procedimento propriamente dito. As três fases experimentais estão descritas na sequência.

Fase 1- Estabelecimento da linha de base. O objetivo dessa fase era verificar a compreensão da tarefa de discriminação condicional auditivo-visual por parte da criança, tendo como estímulo modelo uma palavra ditada e brinquedos conhecidos como estímulos de comparação. Além disso, pretendia-se estabelecer uma linha de base estável de relações entre palavras ditadas e brinquedos familiares, de tal forma que essas relações pudessem ser empregadas como "contraste" familiar nas tentativas de exclusão na fase seguinte. Inicialmente, a pesquisadora dizia: "Vamos fazer uma brincadeira. Eu vou colocar no chão, na sua frente, alguns brinquedos. Eu vou falar o nome de um dos brinquedos e você tem que pegar o brinquedo que eu pedir. Vamos brincar?". No início de cada tentativa a pesquisadora perguntava à criança "Qual desses brinquedos é (e.g., a bola)?”, e esperava-se que ela pegasse $o$ objeto solicitado e o entregasse à pesquisadora. Respostas de seleção corretas eram consequenciadas com frases como: "Muito bom!", ou "Isso mesmo!"; respostas incorretas eram corrigidas ("Não, não é esse. A bola é essa aqui") e, quando tal ocorria, a tentativa era reapresentada. A Fase 1 era constituída por um bloco de seis tentativas, nas quais os modelos auditivos se alternavam (bola, cachorro, macaco, carrinho etc.) e a posição do objeto correto era variável, ao longo das tentativas. O critério de passagem para a fase seguinte foi de seis respostas corretas consecutivas. Caso o critério não fosse atingido, o bloco de tentativas seria reapresentado até que a criança atingisse o critério de tentativas corretas.

Fase 2 - Tentativas de exclusão. A Fase 2 tinha como objetivo verificar se a criança responderia por exclusão diante de um estímulo modelo indefinido (uma palavra desconhecida), selecionando um brinquedo também indefinido. Além disso, como as tentativas de exclusão eram consequenciadas diferencialmente, tinha-se o objetivo de ensinar, por exclusão, as relações entre palavras e objetos indefinidos. A Fase 2 foi constituída por um bloco de oito tentativas, sendo quatro tentativas de linha de base (tentativas com modelo e comparações definidos) e quatro tentativas de exclusão. As tentativas de exclusão eram intercaladas às tentativas de linha de base. $\mathrm{Na}$ primeira tentativa de exclusão, a pesquisadora ditava a palavra "xipite" e, entre as alternativas de escolha, havia brinquedos definidos e um indefinido (I1). Na segunda tentativa de exclusão era ditada a palavra "fulito" e, dentre as alternativas de escolha, havia os brinquedos definidos e os dois indefinidos I1 e I2, dentre os quais a criança deveria selecionar o objeto correspondente à palavra. Na terceira e quarta tentativas de exclusão, as palavras indefinidas "xipite" e "fulito" eram ditadas (uma em cada tentativa) e as alternativas de escolha eram os dois objetos indefinidos, mais dois definidos. Foi considerada uma resposta de exclusão quando a criança, diante da palavra ditada "xipite", selecionou I1 e, diante da palavra ditada "fulito", I2. Todas as respostas das crianças, corretas e incorretas, eram consequenciadas diferencialmente, tanto nas tentativas de linha de base como nas tentativas de exclusão. A seleção do objeto correspondente ao modelo ditado tinha como consequência elogios da 
pesquisadora ("muito bem, é esse mesmo"); quando ocorria a seleção do objeto não correspondente ao modelo, a pesquisadora dizia: "Não, não é esse, pega para mim o X”, repetindo o estímulo modelo. Se, na segunda apresentação do mesmo modelo a criança ainda selecionasse o objeto não correspondente, a pesquisadora repetia o modelo e apontava para o objeto correto, para que a criança o pegasse e entregasse à pesquisadora. $\mathrm{O}$ critério para a criança avançar de fase era a conclusão das oito tentativas, independentemente do número de respostas corretas, ou de respostas corretas após procedimento de correção (importante destacar que todas as respostas incorretas passavam pelo procedimento de correção descrito na Fase 1). A ordem dos objetos diante das crianças era alterada a cada tentativa para que o objeto designado como correto estivesse sempre em uma posição diferente. Além disso, a ordem de introdução dos nomes indefinidos no procedimento foi balanceada entre os participantes: para metade deles o primeiro nome indefinido ditado foi xipite e o segundo fulito; para os demais, a ordem se inverteu.

Fase 3 - Sondas de aprendizagem. O objetivo dessa fase era verificar se, depois das tentativas de exclusão na Fase 2, os participantes relacionavam de forma consistente os nomes "xipite" e "fulito" aos seus objetos correspondentes. Além disso, pretendia-se verificar se as crianças nomeariam corretamente os objetos indefinidos.

Antes de começar a sondagem, a pesquisadora dava a seguinte instrução: "Agora nós vamos continuar a brincadeira, mas eu não vou dizer se você acertou ou errou. Preste bastante atenção na atividade!". Em seguida, eram iniciadas as sondagens de nomeação e emparelhamento ao modelo. Primeiro, foram conduzidas sete tentativas de sonda de nomeação: cada objeto, definido e indefinido, era apresentado individualmente e pedia-se que a criança dissesse o nome do objeto. A pesquisadora pegava de dentro de uma caixa cada um dos objetos e o apresentava à criança, perguntando: "Qual o nome desse brinquedo?”. A criança, então, deveria nomeá-lo. Após a resposta da criança, a pesquisadora recolocava o objeto que estava em sua mão dentro da caixa, pegava outro objeto e o mostrava para a criança, repetindo a pergunta. Esse procedimento foi realizado com os dois objetos indefinidos e cinco definidos.

Logo depois das sondas de nomeação, foram conduzidas as sondas de emparelhamento ao modelo. Nestas, eram ditados, em tentativas distintas, os dois nomes indefinidos ("xipite" e "fulito"), e a criança deveria selecionar o objeto correspondente, tendo como alternativas de escolha I1, I2, e três objetos definidos, que variavam a cada tentativa. O bloco era composto por oito tentativas: quatro tentativas para nomes/objetos definidos e quatro tentativas para nomes/objetos indefinidos (duas para I1 e duas para I2). Assim como na Fase 2, a ordem de introdução dos nomes indefinidos no procedimento foi balanceada entre os participantes. Não havia consequências diferenciais para os desempenhos: após cada resposta de escolha, a pesquisadora redistribuía os brinquedos em frente à criança e dizia "Agora pegue para mim o X", passando, portanto, para a tentativa seguinte. $O$ critério de encerramento dessa fase (e do procedimento) era que a criança nomeasse corretamente os dois objetos indefinidos (I1 e I2) e que os selecionasse corretamente nas quatro sondas de emparelhamento ao modelo (portanto, um critério duplo de aprendizagem desempenhos corretos nas sondas de nomeação e nas quatro sondas de emparelhamento ao modelo).

Caso esse critério duplo de aprendizagem não fosse atingido (i.e., caso o participante acertasse as sondas de emparelhamento ao modelo e não as de nomeação, ou vice-versa, ou se apresentasse apenas desempenho parcialmente correto nas sondas de nomeação e/ou de emparelhamento ao modelo) as Fases 2 e 3 eram reapresentadas até que as crianças nomeassem e selecionassem corretamente os objetos I1 e I2 no mesmo conjunto de sondas da Fase 3, até o limite de 10 reexposições. $\mathrm{Na}$ primeira sessão do procedimento, as crianças poderiam ser expostas até, no máximo cinco reexposições às Fases 2 e 3. Caso o critério duplo de aprendizagem não fosse atingido, uma segunda sessão era conduzida, com, no máximo, outras cinco reexposições às Fases 2 e 3, o que totalizaria o limite de 10 reexposições. As tentativas com os objetos e nomes indefinidos eram consequenciadas na reapresentação da Fase 2, e não consequenciadas na reapresentação da Fase 3.

Deve-se ressaltar que os brinquedos indefinidos apenas eram considerados novos ou desconhecidos em sua primeira apresentação, porém a expressão "indefinido" será mantida a fim de identificálos. Além disso, a rigor apenas a primeira exposição da criança aos nomes e objetos indefinidos poderia ser considerada uma verdadeira tentativa de exclusão (Souza \& Gil, 2018). Porém, para fins de compreensão, todas as tentativas em que os nomes indefinidos foram ditados serão chamadas de tentativas de exclusão. A Tabela 2 apresenta a sequência das fases e as tentativas que foram apresentadas em cada fase, além dos objetos empregados no procedimento.

\section{Análise dos dados}

Uma segunda observadora fez um registro independente das respostas das crianças de cerca de $30 \%$ das filmagens das sessões experimentais e esses dados foram comparados com o registro feito pela pesquisadora. O índice de concordância (Danna \& Matos, 2015) foi de $98 \%$. Foi calculada a porcentagem de acertos dos participantes (e dos grupos) nas tentativas de exclusão (Fase 2) e nas sondas de nomeação e emparelhamento ao modelo (Fase 3 ). O cálculo levou em conta o número de tentativas que cada criança realizou até atingir o critério de encerramento do procedimento (ou até serem completadas as 10 reexposições às Fases 2 e 3). A porcentagem média de acertos dos grupos foi calculada pela média das porcentagens individuais dos participantes de cada grupo em cada tarefa. 
Tabela 2.

Linha de Base, Tentativas de Exclusão, Sondas de Aprendizagem.

\begin{tabular}{lccc}
\hline $\begin{array}{l}\text { Fase do } \\
\text { procedimento }\end{array}$ & $\begin{array}{c}\text { Estímulo modelo } \\
\text { (palavra ditada) }\end{array}$ & Estímulos de comparação & $\begin{array}{l}\text { Resposta } \\
\text { esperada }\end{array}$ \\
\hline
\end{tabular}

Fase 2 -

Tentativas de

Exclusão "xipite"

D1, D2, D3, D4, D5
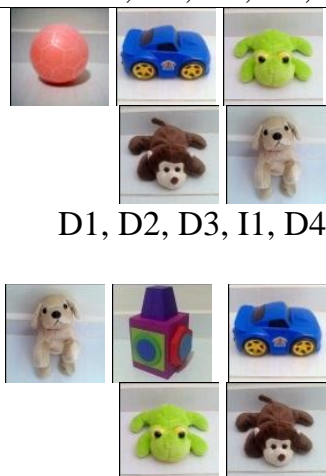

D

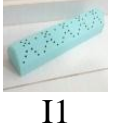

I1

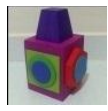

I2

D5, I2, D2, I1, D3, D4

Fase 3 -

Sondas de

Nomeação

"Qual é o nome desse
brinquedo?" (D)

"Qual é o nome desse brinquedo?" (I)

Sondas de

Emparelhamento ao Modelo "xipite"

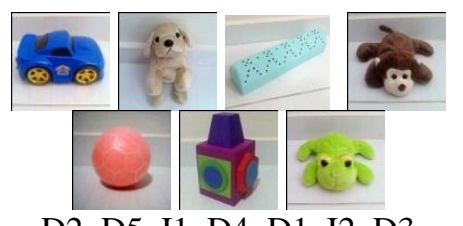

D2, D5, I1, D4, D1, I2, D3

"fulito"

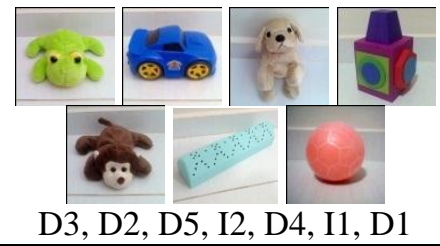

Nome de D1, D2 e D3.

Nome de I1 e

I2.
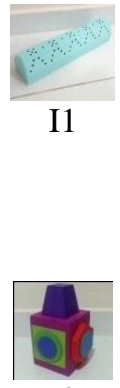

I2

Legenda: D: definido, I: indefinido. 


\section{RESULTADOS}

De modo geral, o desempenho das crianças com DT e com AF foram muito semelhantes. Todas as crianças com DT e com AF responderam por exclusão na apresentação inicial das novas palavras e objetos indefinidos, apesar de terem apresentado erros nas tentativas subsequentes de exclusão, especialmente com a introdução da segunda nova relação. Além disso, todas as crianças, dos dois grupos, responderam corretamente às sondas de emparelhamento ao modelo das duas relações palavra-objeto até o quinto bloco de exposições (com exceção de uma criança DT, que o fez no sexto bloco), mas apenas seis nomearam corretamente os brinquedos (três de cada grupo).
A maioria dos participantes (10 do grupo DT e seis do grupo $\mathrm{AF}$ ) realizou 22 tentativas de exclusão (duas por bloco), 22 sondas de emparelhamento ao modelo (duas por bloco) e 11 sondas de nomeação, de cada relação ensinada/testada, completando o número máximo de reexposições (10, além do bloco inicial). Três participantes (DT9, AF17 e AF19) realizaram um número menor de tentativas por terem atingido os dois critérios de aprendizagem (selecionar e nomear corretamente os dois estímulos indefinidos no mesmo bloco). Todos os participantes acertaram $100 \%$ das tentativas da linha de base. Os resultados dos participantes de ambos os grupos nas tentativas de exclusão, sondas de emparelhamento ao modelo e de nomeação são apresentados na Figura 1.

\section{Participantes do DT}
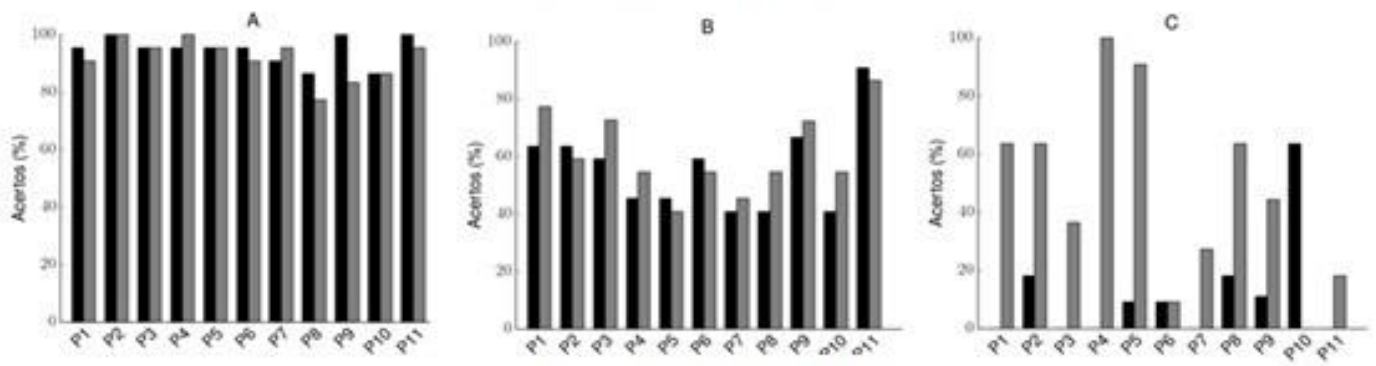

Participantes com AF
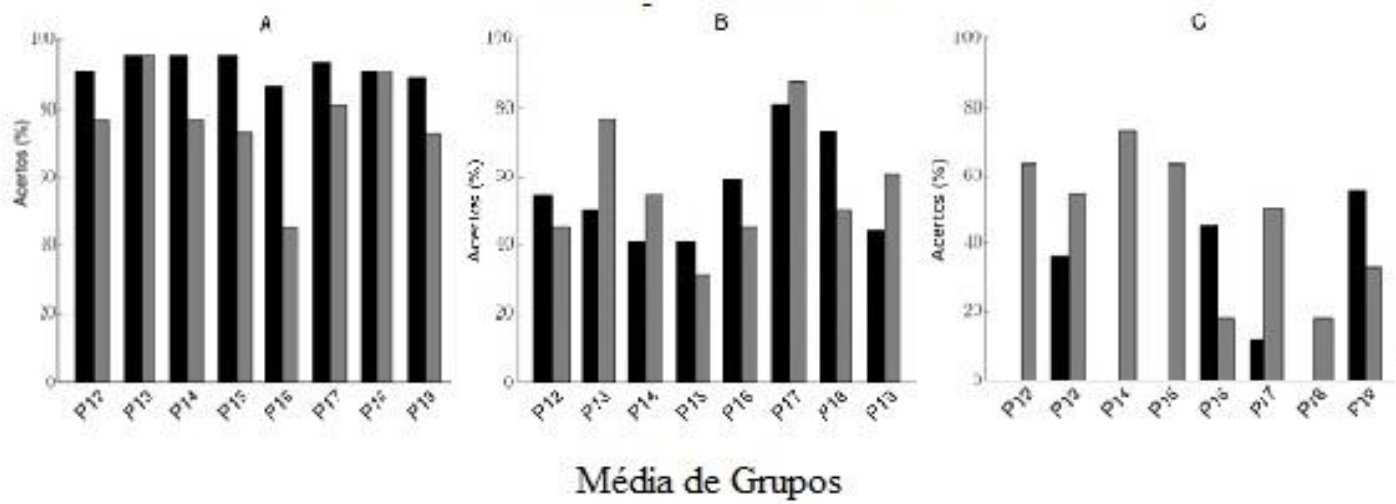

Média de Grupos

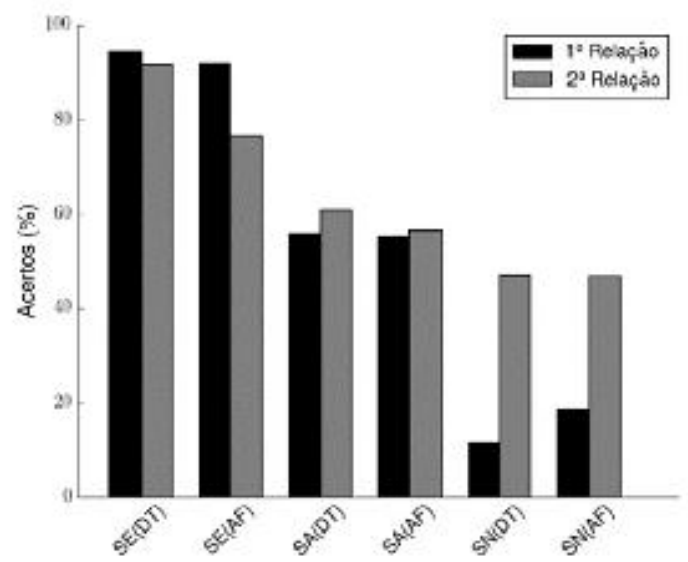

Figura 1. Porcentagem de acertos dos participantes dos grupos DT (desenvolvimento típico) e AF (atraso da fala) nas tentativas de Exclusão (A), Emparelhamento ao modelo (B) e de Nomeação (C). O painel inferior (Média de grupos) apresenta a porcentagem média de acertos dos grupos DT e AF nas Tentativas de Exclusão (SE), Sondas de Emparelhamento ao modelo (SA) e Nomeação $(\mathrm{SN})$. Barras escuras referem-se à primeira relação ensinada e barras em cinza, à segunda relação. 
Todos os participantes do grupo DT apresentaram mais de $80 \%$ de acertos nas tentativas de exclusão com as duas relações indefinidas (painel superior A), ainda que todos os participantes tenham apresentado erros, tanto nas tentativas envolvendo a primeira relação, como a segunda.

No grupo AF, três dos oito participantes apresentaram porcentagens de acerto acima de $80 \%$ nas duas relações nas tentativas de exclusão (painel inferior A, Figura 1 - AF13, AF17 e AF18), um número menor de participantes do que no grupo DT. Os demais participantes atingiram mais de $80 \%$ de acertos apenas na primeira relação ensinada, e cerca de $70 \%$ de acertos na segunda relação, exceto AF16 cujo desempenho na segunda relação foi de cerca de $40 \%$ de acertos.

Nas sondas de emparelhamento ao modelo (painéis B), em que a tarefa do participante era emparelhar o modelo auditivo indefinido ditado ao objeto indefinido correspondente, metade dos participantes do grupo DT apresentou entre 50 e $70 \%$ de acertos nas duas relações, e DT11 apresentou mais de $80 \%$ de acertos para as duas relações. Os demais apresentaram em torno de 40 e $50 \%$ de acertos. O desempenho dos participantes do grupo AF foi mais variável do que o do grupo DT: apenas AF17 apresentou mais de $80 \%$ de acertos nas duas relações; AF13 e AF18 apresentaram entre 50 e $70 \%$ de acertos, AF12, AF14, AF 16 e AF19 apresentaram entre 40 e $50 \%$ de acertos, e AF15 menos de $40 \%$ nas duas relações.

Nas sondas de nomeação, os desempenhos do grupo DT foram variáveis (painel superior C). Todos os participantes emitiram nomeações corretas para ao menos um dos objetos; DT2, DT5, DT6, DT8 e DT9 para os dois objetos, sendo que DT2, DT5 e DT9 no mesmo bloco de sondagem. Observa-se a tendência de maior emissão do nome indefinido da segunda relação apresentada para todos os participantes, exceto DT10. No grupo AF todos os participantes emitiram ao menos um dos dois nomes indefinidos (em geral o segundo apresentado), e quatro emitiram os dois nomes corretamente (painel inferior $\mathrm{C}$ ), sendo que AF13, AF17 e AF19 no mesmo bloco de sondagem.

Apesar das diferenças de desempenho individual apontadas nos painéis $\mathrm{A}, \mathrm{B}$ e $\mathrm{C}$, as médias dos grupos $\mathrm{AF}$ e DT (painel inferior da Figura 1) indica que os desempenhos dos grupos DT e AF foram bastante similares nas tentativas de exclusão, sondas de emparelhamento ao modelo e nomeação: alta porcentagem de acertos nas tentativas de exclusão (com pequena perturbação na média de acertos do grupo AF para a segunda relação), em torno dos $50 \%$ de acertos nas sondas de emparelhamento ao modelo, e baixa porcentagem de nomeações corretas, que ocorreram, sobretudo, para a segunda relação ensinada.

A média dos desempenhos grupais apresenta apenas os dados de cada grupo a partir do total de reexposições às tentativas de exclusão, que foi variável entre os participantes. Ao se analisar o desempenho dos participantes individuais ao longo das sondagens, é possível observar que todas as crianças, ao longo do procedimento, atingiram o critério de acertos estabelecidos para as sondas de emparelhamento ao modelo. No entanto, apenas seis crianças (três de cada grupo) atingiram o critério de acertos nas nomeações, todas após a sexta exposição às tentativas do tipo exclusão. Portanto, apesar de emparelharem corretamente os modelos auditivos aos objetos correspondentes, $72 \%$ de crianças DT e $62 \%$ de crianças AF não apresentaram nomeação correta dos dois objetos indefinidos, mesmo após a exposição a 10 tentativas de exclusão. DT11, por exemplo, acertou todas as sondas de emparelhamento ao modelo desde a primeira exposição às tentativas de exclusão, mas não nomeou corretamente os dois estímulos (ver Tabela 3).

Outra característica observada no padrão de responder das crianças nas sondas de emparelhamento ao modelo foi a instabilidade na manutenção desse desempenho. Como o critério de encerramento do procedimento era duplo (acertos nas sondas de emparelhamento ao modelo e nas de nomeação), se o critério de acertos na nomeação não era alcançado, a reexposição às tentativas de exclusão e às sondas de emparelhamento ao modelo era mantida. Observou-se que a maioria das crianças dos dois grupos apresentou erros nas sondas de emparelhamento ao modelo, mesmo após uma sequência de três ou quatro blocos de acertos consecutivos.

A Tabela 3 apresenta as respostas dos participantes nas sondas de nomeação. De modo geral, as crianças do grupo DT apresentaram mais respostas às sondas de nomeação (ainda que incorretas) que as crianças do grupo AF. Além disso, a maioria dos participantes dos dois grupos tenderam a dizer o segundo nome apresentado nas tentativas de exclusão (e.g., DT1, DT2, DT4, DT5, DT8, AF12, AF14, AF15 e AF17), e alguns desses foram os que nomearam corretamente ambos os objetos. Apenas DT10 tendeu a nomear corretamente apenas o primeiro objeto indefinido apresentado, utilizando esse mesmo nome (de forma incorreta) para o outro objeto em um número reduzido de sondas. Com exceção de DT11 e AF17, todas as crianças, ao nomearem os objetos indefinidos, ainda que de forma incorreta, emitiram os nomes indefinidos empregados no procedimento e não outros nomes definidos.

\section{DISCUSSÃO}

Os resultados aqui relatados não evidenciaram relação entre a condição de desenvolvimento da linguagem dos participantes (DT ou AF) e a aprendizagem das relações nome-objeto, uma vez os desempenhos dos dois grupos se mostraram bastante semelhantes. Dessa forma, não se confirmou a hipótese de que crianças com AF precisariam de um número maior de exposições às tentativas de exclusão para aprender duas relações nome-objeto, em comparação com crianças DT, além de não terem sido verificadas dificuldades específicas do grupo AF para responder por exclusão. Esses resultados não confirmam os do estudo de Ellis Weismer et al. (2013), que sugeriu que crianças com AF teriam dificuldades de responder por exclusão e aprender novas relações entre palavras e objetos via exclusão. 
Tabela 3.

Respostas dos Participantes do Grupo com DT e Grupo com AF às Sondas de Nomeação. Ao Lado de Cada Participante a Ordem em que Foram Apresentados os Objetos Indefinidos ao Longo do Procedimento.

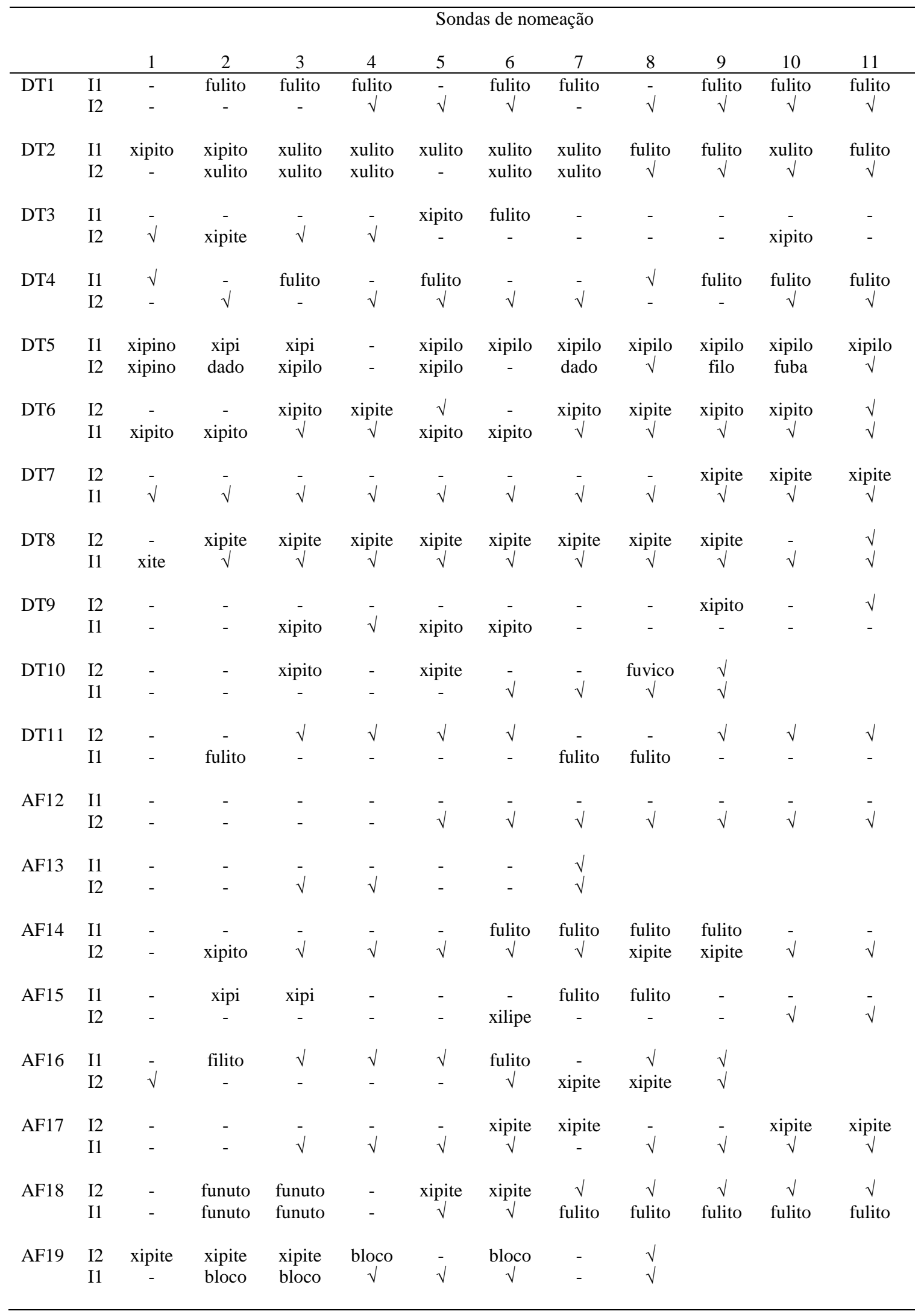

Nota: $\mathrm{P}$ = participante; I = objeto indefinido; I1 = "xipite"; I2 = "fulito"; - = ausência de resposta; $\sqrt{\text { = }}$ resposta correta. 
Fonseca (2016), comparando o desempenho de crianças com e sem AF em tarefas de exclusão envolvendo substantivos e verbos também não encontrou diferenças estatisticamente significativas no desempenho por exclusão dos dois grupos. Apesar de Fonseca (2016) não ter sondado a aprendizagem de relações entre palavras e figuras/vídeos de ações, seus participantes (crianças entre 3 e 4 anos) não apresentaram desempenhos diferentes em função de seu desenvolvimento de fala.

Os resultados do presente estudo e do de Fonseca (2016) também são semelhantes a estudos realizados sobre o desempenho por exclusão de crianças com distúrbios específicos de linguagem, nos quais o desempenho compreensivo dessas crianças em tarefas de mapeamento rápido não foi diferente do de crianças com desenvolvimento típico (Dollaghan, 1987; Eyer et al., 2002; Gray, 2005). No conjunto, verifica-se, portanto, que o desempenho por exclusão é robusto mesmo em crianças com atraso da fala ou com outros tipos de atraso de desenvolvimento (e.g., Costa, McIlvane, Wilkinson, \& de Souza, 2001), e que as dificuldades de ampliação de vocabulário de crianças com $\mathrm{AF}$ aparentemente não podem ser atribuídas a dificuldades específicas no desempenho por exclusão. É possível que a diferença encontrada nos desempenhos por exclusão entre crianças com AF e com DT em outros estudos tenha sido decorrente de características específicas desses procedimentos, tal como discutido por Souza et al. (2018). De modo geral, os estudos sobre mapeamento rápido não estabelecem inicialmente uma linha de base estável de discriminações condicionais com nomes e objetos conhecidos. Após duas ou três apresentações de nomes e figuras/brinquedos familiares às crianças, os estudos apresentam as tentativas de teste (nomes e objetos desconhecidos). A falta de um desempenho de linha de base estável pode ser responsável por eventuais dificuldades na tarefa de responder por exclusão, mas essa é apenas uma hipótese a ser melhor investigada em estudos posteriores.

Outro fator de interesse verificado nos resultados refere-se à aprendizagem dos desempenhos de emparelhamento ao modelo e de nomeação com os estímulos indefinidos. Todas as crianças aprenderam a emparelhar as duas figuras indefinidas aos seus respectivos nomes em seis exposições ou menos. Esse resultado pode estar relacionado ao procedimento de ensino adotado - a introdução sucessiva de estímulos (Wilkinson et al., 2003): a apresentação conjunta dos dois estímulos indefinidos já nas tentativas de ensino parece favorecer a discriminação entre eles, ainda que piore os resultados de exclusão das crianças na introdução da segunda relação indefinida.

Resultados semelhantes foram encontrados por Ribeiro e Schmidt (2015), em um procedimento de introdução sucessiva de estímulos para ensino de duas relações nome-objeto para bebês entre 17 e 20 meses. A introdução da segunda relação nome-objeto desestabilizou o desempenho dos bebês nas tentativas de ensino por exclusão, mas, nos testes, eles apresentaram indícios de aprendizagem dessas relações, apesar de não terem atingido o critério estabelecido. É preciso considerar que o presente estudo avança nos resultados obtidos por Ribeiro e Schmidt (2015), uma vez que, aqui, todas as crianças atingiram o critério nas sondas de aprendizagem. Comparando os dois estudos, observa-se que as crianças do presente estudo, além de serem mais velhas, também foram expostas às tentativas de ensino por um número maior de tentativas: enquanto que em Ribeiro e Schmidt (2015) as crianças eram expostas a cinco tentativas de ensino para cada relação, no presente estudo, antes de cada bloco de sondas as crianças eram expostas a duas tentativas de ensino para cada relação, o que significa que, no quinto bloco de sondagem, as crianças já haviam sido expostas a 10 tentativas de ensino. O presente estudo sugere, então, que o número de tentativas de ensino é uma variável importante em procedimentos de ensino de relações entre palavras e figuras/objetos.

Estudos que investigavam a quantidade de exposição às tentativas de exclusão necessária para aprendizagem de relações entre palavras e seus referentes registraram um número entre duas e 10 exposições para que participantes de diferentes idades e perfis de desenvolvimento emparelhassem corretamente a palavra indefinida ao estímulo visual correspondente (Costa et al, 2013; Langsdorff, et al., 2017; Langsdorff, et al., 2015; Souza et al., 2016). Essa variabilidade foi encontrada mesmo entre participantes com idades e perfis de desenvolvimento semelhantes, indicando que variáveis individuais específicas, ainda não investigadas, podem facilitar ou dificultar essa aprendizagem, o que parece ser um caminho de investigação importante para pesquisas futuras.

Porém, ainda que todas as crianças do presente estudo, independentemente de terem DT ou AF, tenham atingido o critério estabelecido nas sondas de emparelhamento ao modelo, apenas seis das 19 crianças apresentaram nomeação correta dos dois objetos indefinidos. Algumas pesquisas têm investigado a nomeação de estímulos visuais a partir de tentativas de ensino de relações nome-objeto por exclusão, com resultados variáveis (Costa et al, 2013; Langsdorff, et al.,2017; Langsdorff, et al., 2015; Souza et al, 2016). Souza et al. (2016), por exemplo, não observaram resultados consistentes de nomeação ao estudarem crianças entre 27 e 36 meses, mas o estudo tinha como foco o desempenho correto nas sondas de emparelhamento ao modelo. Langsdorff et al. (2015 e 2017) verificaram aprendizagem do desempenho de nomeação após tentativas de ensino por exclusão, mas os participantes desses estudos eram mais velhos que os da presente pesquisa e aprenderam uma única relação nome-objeto por vez.

O estudo de Costa et al. (2013) tem maior similaridade com o aqui descrito. Nele, oito crianças aprenderam duas relações nome-figura e nomearam os estímulos novos com no mínimo 3 e no máximo 10 reexposições. No presente estudo, as seis crianças que nomearam os dois objetos o fizeram após no mínimo sete e no máximo 10 reexposições. Assim, os resultados obtidos nesta pesquisa não são compatíveis com os 
apresentados por Costa et al. (2013). Os motivos para a discrepância encontrada nos resultados dos dois estudos ainda não estão claros. Algumas possibilidades, porém, podem ser levantadas. O estudo de Costa et al. (2013) foi conduzido por meio de um computador, de forma mais rápida e em uma única sessão, enquanto que todas as crianças do presente estudo precisaram de duas sessões para concluir o procedimento. A condução do experimento no computador pode ter melhorado o engajamento das crianças nas tarefas, mantendo-as mais atentas aos estímulos apresentados, favorecendo o desempenho dos participantes. Além da interrupção da sessão, que poder ter produzido uma interferência no desempenho das crianças do presente estudo (inclusive porque o intervalo entre as sessões foi de até uma semana), é possível que a repetição dos blocos de ensino tenha produzido uma redução do engajamento delas na tarefa. Essa hipótese se sustenta, inclusive, pela instabilidade do desempenho de algumas crianças nas sondas de emparelhamento ao modelo. Uma discussão sobre a estabilidade das relações emergentes após exclusão pode ser verificada em Souza e Gil (2018).

Apesar do número reduzido de crianças que apresentaram nomeação consistente no presente estudo, em comparação ao estudo de Costa et al. (2013), os resultados aqui apresentados são de grande interesse pela ausência de diferenças entre os dois grupos, o que pode sugerir que o procedimento aqui empregado pode ser utilizado também com crianças com atraso da fala, ainda que a questão sobre a emergência de desempenho de nomeação após a aprendizagem de desempenho de emparelhamento ao modelo permaneça em discussão.

Várias pesquisas indicam que o responder por exclusão e a aprendizagem de relações nome-objeto/figura a partir de poucas tentativas de exclusão em sondas de emparelhamento ao modelo, não são condições suficientes para a nomeação dessas figuras/objetos (e.g., Bandini, Sella, Postalli, Bandinni, \& Silva, 2012; Schmidt et al., 2016). O desempenho de nomeação não é uma decorrência direta de tentativas de exclusão: o desempenho de emparelhamento ao modelo e a nomeação são repertórios independentes (de ouvinte e de falante), tratando-se, portanto, de comportamentos verbais distintos (Greer \& Du, 2015), com pré-requisitos também distintos.

A nomeação pode ser considerada uma behavioral cusp ou "cunha comportamental" (RosalesRuiz \& Baer, 1996), que começa a ocorrer aos 2 ou 3 anos de idade (Greer \& Longano, 2010), e que possibilita à criança aprender com o ambiente de uma forma que ela não conseguia antes. Greer e Du (2015) afirmam que a emergência de nomeação a partir de tentativas de exclusão, especificamente, é uma cunha comportamental à parte. Ao nomear um objeto, propriedade de um objeto ou uma ação a partir de uma situação em que o responder por exclusão é possível, a criança se torna capaz de nomear um número cada vez maior de elementos do mundo, sem a necessidade de ensino direto. No entanto, os mesmos autores demonstram que crianças que apresentam nomeação não necessariamente apresentam nomeaçãopor-exclusão, o que pode ser ensinado por meio de procedimentos de exclusão. Entender as condições necessárias e suficientes para que a nomeação por exclusão ocorra é ainda um desafio para pesquisadores da área e demanda esforços de pesquisa, por ser um tópico com grande aplicabilidade prática em diferentes ambientes e para populações com diferentes perfis de desenvolvimento.

Futuras pesquisas devem avançar em procedimentos que favoreçam a aprendizagem de diferentes repertórios verbais em crianças, reunindo informações derivadas de estudos básicos para ensino de diferentes operantes verbais, tanto em ambientes planejados, como em ambientes naturais. Atrasos de vocabulário têm sido referidos como uma situação de risco para a aprendizagem de variados repertórios acadêmicos (e.g., França, Wolff, Moojen, \& Rotta, 2004), e procedimentos que possam ser aplicados em ambientes escolares para crianças com menos de 5 anos são uma necessidade premente na nossa sociedade (Löhr \& Gil, 2015).

\section{DECLARAÇÃO DE CONFLITO DE INTERESSES}

As autoras declaram que não há qualquer conflito de interesses relativos à publicação deste artigo.

\section{CONTRIBUIÇÃO DE CADA AUTORA}

Fernanda Marques Rezende participou da concepção da pesquisa, coleta e análise de dados, bem como de redação do manuscrito. Julia Fonseca participou da concepção da pesquisa e da coleta de dados. Andréia Schmidt participou na concepção da pesquisa, supervisão da coleta e análise de dados e redação do manuscrito.

\section{DIREITOS AUTORAIS}

Este é um artigo aberto e pode ser reproduzido livremente, distribuído, transmitido ou modificado, por qualquer pessoa desde que usado sem fins comerciais. $\mathrm{O}$ trabalho é disponibilizado sob a licença Creative Commons 4.0 BY-NC.

\section{(cc) $\mathrm{BY}-\mathrm{NC}$}

\section{REFERÊNCIAS}

Bandini, C. S. M., Sella, A. C., Postalli, L. M. M., Bandini, H. H. M., \& Silva, E. T. P. (2012). Effects of selection tasks on naming emergence in children. Psicologia: Reflexão e Crítica, 25(3), 568-577. doi: 10.1590/S0102-79722012000300017

Capovilla, F. C., Negrão, V. D. \& Damázio, M. (2011). Teste de vocabulário por figuras USP-Tvfusp.

Cavalheiro, L. G., Brancalioni, A. R., \& Keske-Soares, M. (2012). Prevalência do desvio fonológico em crianças da cidade de Salvador, Bahia. Revista da Sociedade Brasileira de Fonoaudiologia, 17(4), 441-446. doi: 10.1590/S1516-80342012000400013

Costa, A. R. A., de Rose, J. C. C., \& de Souza, D. G. (2010). Interferência de variáveis de contexto em sondas de exclusão com substantivos e verbos novos. Acta Comportamentalia, 18(1), 35-54.

Costa, A. R. A., Grisante, P. C., Domeniconi, C., de Rose, J. C. C., \& de Souza, D. G. (2013). Nomeação de 
estímulos novos a partir da seleção por exclusão. Paidéia, 23(55), 217-224. doi: 10.1590/198243272355201309

Costa, A. R. A., McIlvane, J. W., Wilkinson, K. M., \& de Souza, D. G. (2001). Emergent word-object mapping by children: Further studies using the blank comparison technique. The Psychological Record, 51, 343-355.

Dixon, L. S. (1977). The nature of control by spoken words over visual stimulus selection. Journal of the Experimental Analysis of Behavior, 27, 433-442. doi: 10.1901/jeab.1977.27-433

Dollaghan, C. A. (1987). Fast mapping in normal and language-impairment children. Journal of Speech and Hearing Disorders, 52, 218-222. doi:10.1044/jshd.5203.218

Domeniconi, C., Costa, A. R. A., de Souza, D. G., \& de Rose, J. C. C. (2007). Responder por exclusão em crianças de 2 a 3 anos em uma situação de brincadeira. Psicologia: Reflexão e Crítica, 20(2), 342-350.

Ellis Weismer, S., Venker, C. E., Evans, J. L., \& Moyle, M. J. (2013). Fast mapping in late-talking toddlers. Applied Psycholinguistic, 34(1), 69-89. doi: 10.1017/S0142716411000610

Eyer, J. A., Leonard, L. B., Bedore, L. M., McGregor, K. K., Anderson, B., \& Viescas, R. (2002). Fast mapping of verbs by children with specific language impairment. Clinical Linguistics \& Phonetics, 16, 5977. doi: 10.1080/02699200110102269

Fonseca, J. (2016). Responder por exclusão em crianças com atraso de linguagem. Dissertação de Mestrado, Faculdade de Filosofia, Ciências e Letras de Ribeirão Preto, Universidade de São Paulo, Ribeirão Preto.

França, M. P., Wolf, C. L., Moojen, S., \& Rotta, N. T. (2004). Aquisição da linguagem oral: Relação e risco para a linguagem escrita. Arquivos de NeuroPsiquiatria, 62(2b), 469-472. doi: 10.1590/S0004282X2004000300017.

Goulart, B. N. G. D., \& Chiari, B. M. (2007). Prevalence of speech disorders in school children and its associated factors. Revista de Saúde Pública, 41(5), 726-731. doi: 10.1590/S0034-89102007000500006

Gray, S. (2005). Word learning by preschoolers with specific language impairment: Effect of phonological or semantic cues. Journal of Speech, Language, and Hearing Research, 48, 1452-1467. doi: 10.1044/10924388(2005/101)

Greer, R. D., \& Du, L. (2015). Experience and the onset of the capability to learn names incidentally by exclusion. The Psychological Record, 65, 355-373. doi: 10.1007/s40732-014-0111-2

Greer, R. D., \& Longano, J. (2010). A rose by naming: How we may learn how to do it. The Analysis of Verbal Behavior, 26, 73-106. Doi: 10.1007/BF03393085

Hage, S. R. V., \& Guerreiro, M. M. (2010). Distúrbio específico de linguagem: Aspectos linguísticos e neurobiológicos. In F. D. M. Fernandes, L. P. Ferreira, D. M. Befi-Lopes, \& S. C. O. Limongi (Orgs.),
Tratado de fonoaudiologia (pp. 323-329). São Paulo: Editora Roca.

Langsdorff, L. C., Domeniconi, C., Schmidt, A., Gomes, C. G., \& de Souza, D. G. (2017). Learning by exclusion in individuals with autism and Down syndrome. Psicologia: Reflexão e Crítica, 30, 9. doi: 10.1186/s41155-017-0064-X

Langsdorff, L. C., Schmidt, A., \& Domeniconi, C. (2016). Aprendizagem de relações auditivo-visuais por meio de tentativas de exclusão. Interação em Psicologia, 19(1), 25-35. doi: 10.5380/psi.v19i1.33978

Löhr, T., \& Gil, M. S. C. A. (2015). Learning by playing: Echo and tact in expanding the verbal repertoire of infants. Paidéia, 25(60), 77-84. doi: 10.1590/198243272560201510

Rescorla, L. (2002). Language and reading outcomes to age 9 in late-talking toddlers. Journal of Speech, Language, and Hearing Research, 45(2), 360-371. doi: 10.1044/1092-4388(2002/028)

Rescorla, L. (2009). Age 17 language and reading outcomes in late-talking toddlers: Support for a dimensional perspective on language delay. Journal of Speech, Language, and Hearing Research, 52(1), 1630. doi: 10.1044/1092-4388(2008/07-0171)

Ribeiro, J., \& Schmidt, A. (2015). Aprendizagem de relações palavra-objeto por bebês em um procedimento de introdução sucessiva de estímulos. Revista Brasileira de Análise do Comportamento, 11(1), 7079. doi: 10.18542/rebac.v11i1.3777

Rosales-Ruiz, J., \& Baer, D. M. (1997). Behavioral cusps: A developmental and pragmatic concept for behavior analysis. Journal of Applied Behavior Analysis, 30, 533-544. doi: 10.1901/jaba.1997.30-533

Schmidt, A., Franco, M., Lotério, L. S., \& Gomes, G. F. (2016). Learning name-object relations after a single exclusion trial in 18- to 48-month-old children. The Psychological Record, 66(1), 53-63. doi: 10.1007/s40732-015-0151-2

Skinner, B. F. (1957). Verbal Behavior. New York: Applenton-Century-Crofts.

Souza, L. M. R. D., \& Gil, M. S. C. A. (2018). Estabelecimento de relações emergentes nome-objeto por bebês de 18 a 22 meses. Acta Comportamentalia, 26(2), 199-215.

Souza, L. M. R. D., Gil, M. S. C. A., \& Garcia, L. T. (2018). Learning by exclusion in toddlers. Paidéia, 28, e2810. doi: 10.1590/1982-4327e2810

Souza, L. M. R., Sousa, N. M., \& Gil, M. S. C. A. (2016). Aprendizagem de relações emergentes por exclusão em crianças de 2 a 3 anos. Revista Brasileira de Análise do Comportamento, 12(1), 44-53. doi: 10.18542/rebac.v12i1.3788

Wilkinson, K. M., \& McIlvane, W. J. (1997). Blank comparison analysis of emergent symbolic mapping by young children. Journal of Experimental Child Psychology, 67, 115-130. doi: 10.1006/jecp.1997.2402

Wilkinson, K. M., Ross, E., \& Diamond, A. (2003). Fast mapping of multiple words: Insights into when "the information provided" does and does not equal "the information perceived". Applied Developmental 
Psychology, 24, 739-762. doi: 10.1016/j.appdev.2003.09.006

Zubrick, S. R., Taylor, C. L., Rice, M. L., \& Slegers, D. W. (2007). Late language emergence at 24 months: An epidemiological study of prevalence, predictors, and covariates. Journal of Speech, Language, and Hearing Research, 50(6), 1562-1592. doi: 10.1044/10924388(2007/106)

Submetido em: 27/02/2018 Aceito em: 09/11/2018 